\title{
COMPACT COMPLEX THREEFOLDS OF CLASS $L$ ASSOCIATED TO POLYNOMIAL AUTOMORPHISMS OF $\mathbb{C}^{3}$
}

\author{
Karl Oelueklaus and Julie Renaud
}

Abstract

We construct new families of non-Kähler compact complex threefolds belonging to Kato's Class $L$. The construction uses certain polynomial automorphisms of $\mathbb{C}^{3}$. We also study basic properties of our manifolds.

\section{Introduction and Construction}

We start by recalling the definition of Kato's Class $L$, see [6]. Let

$$
V:=\left\{\left.\left[z_{0}: z_{1}: z_{2}: z_{3}\right] \in \mathbb{P}_{3}(\mathbb{C})|| z_{0}\right|^{2}+\left|z_{1}\right|^{2}>\left|z_{2}\right|^{2}+\left|z_{3}\right|^{2}\right\} .
$$

A (not necessarily compact) irreducible complex space $X$ is said to be of Class L, if and only if $X$ contains an open subset biholomorphic to $U$. M. Kato studied several interesting (smooth) examples and properties of Class $L$ spaces, see $[\mathbf{6}]$ and its references. The aim of the present paper is to construct new families of examples of non-Kähler class $L$ spaces and threefolds with fundamental group $\mathbb{Z}$. All previously known examples of such manifolds where quotients of the complement of two disjoint linear rational curves in $\mathbb{P}_{3}(\mathbb{C})$, which all admit projective structures (see [7]).

In order to simplify the approach, we give the construction and study the properties in a special case. In the last section we give all quadratic automorphisms of $\mathbb{C}^{3}$ which allow exactly the same arguments.

Consider the following polynomial automorphism $H$ of $\mathbb{C}^{3}$

$$
H(x, y, z)=\left(\begin{array}{c}
x^{2}+c y^{2}+z \\
y^{2}+x \\
y
\end{array}\right),
$$

2000 Mathematics Subject Classification. Primary: 32J15.

Key words. Polynomial automorphisms of $\mathbb{C}^{3}$, Global spherical shell, Kato's Class $L$. 
the constant $c \in \mathbb{C}$ being chosen arbitrarily. The inverse automorphism is given by

$$
H^{-1}(x, y, z)=\left(\begin{array}{c}
y-z^{2} \\
z \\
x-\left(y-z^{2}\right)^{2}-c z^{2}
\end{array}\right) .
$$

We extend $H$ and $H^{-1}$ to $\mathbb{P}_{3}=\{[x: y: z: t]\}$ and, keeping the same notations, we get:

$$
\begin{aligned}
H[x: y: z: t] & =\left[x^{2}+c y^{2}+z t: y^{2}+x t: y t: t^{2}\right] \\
H^{-1}[x: y: z: t] & =\left[y t^{3}-z^{2} t^{2}: z t^{3}: x t^{3}-\left(y t-z^{2}\right)^{2}-c z^{2} t^{2}: t^{4}\right] .
\end{aligned}
$$

The set of indeteminacy of $H$ is given by

$$
I^{+}=\{x=y=t=0\}
$$

which is the point $[0: 0: 1: 0]$, and that of $H^{-1}$ equals to

$$
I^{-}=\{z=t=0\}
$$

which is the projective line $\{[x: y: 0: 0]\}$.

It is clear that $I^{+} \cap I^{-}=\emptyset$, i.e. automorphism $H$ is regular in the sense of Sibony $[\mathbf{8}]$.

Definition 1.1. Let $M$ be a metric space and $f: M \rightarrow M$ a continous mapping. A compact subset $K \subset M$ is called attractor for $f$ if there is an open neighbourhood $V \subset M$ of $K$ such that $f(V) \Subset V$ and $\bigcap_{n \geq 0} f^{n}(V)=K$.

We have the following

Proposition 1.2 (see [8]). Let $f$ be a regular polynomial automorphism of $\mathbb{C}^{k}$ of algebraic degree $d \geq 2$ considered as a birational map of $\mathbb{P}_{k}(\mathbb{C})$. Then the set of indeterminacy $I^{+}$of $f$ is an attractor for $f^{-1}$ and, conversely, the set of indeterminacy $I^{-}$of $f^{-1}$ is an attractor for $f$.

The hyperplane at infinity minus the indeterminacy set $\{t=0\} \backslash I^{+}$ is mapped by $H$ to the projective line

$$
X^{+}:=I^{-}=\{z=t=0\}=\{[x: y: 0: 0]\}
$$

which is an attractor for $H$.

Let

$$
U^{+}:=\left\{w \in \mathbb{C}^{3}, \lim _{n \rightarrow \infty} H^{n}(w) \in X^{+}\right\}
$$

be the bassin of attraction of $H$ in $\mathbb{C}^{3}$. 
The automorphism $H$ being algebraic, it follows that its graph $\operatorname{gr}(H) \subset$ $\mathbb{C}^{3} \times \mathbb{C}^{3}$ has the property that its topological closure $W:=\overline{\operatorname{gr}(H)} \subset$ $\mathbb{P}_{3} \times \mathbb{P}_{3}$ is a (not necessarily smooth) projective variety. We have the following commutative diagram

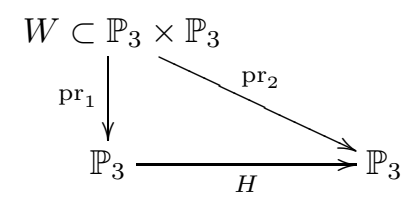

where $\operatorname{pr}_{i}, i=1,2$ are the projections to the $\mathbb{P}_{3}$-factors. In the following we denote by $p_{i}$ the restrictions of $\operatorname{pr}_{i}$ to $W, i=1,2$.

An easy calculation gives

$$
W=\operatorname{gr}(H) \cup D_{1} \cup D_{2}
$$

with

$$
\begin{aligned}
D_{1}:=\left\{\left[z_{0}: z_{1}: z_{2}: 0\right],\left[z_{0}^{2}+c z_{1}^{2}: z_{1}^{2}: 0: 0\right]\right\} \\
\cup\left\{[0: 0: 1: 0],\left[y_{0}: y_{1}: 0: 0\right]\right\}, \\
D_{2}:=\left\{[0: 0: 1: 0],\left[y_{0}: y_{1}: y_{2}: 0\right]\right\} .
\end{aligned}
$$

For the intersection we have $l:=D_{1} \cap D_{2}=\left\{[0: 0: 1: 0],\left[y_{0}: y_{1}: 0: 0\right]\right\}$.

Let $\varepsilon>0$ and $V_{\varepsilon}$ the neighbourhood of $X^{+}$in $\mathbb{P}_{3}$ of the form

$$
V_{\varepsilon}:=\left\{\left.\left[z_{0}: z_{1}: z_{2}: z_{3}\right] \in \mathbb{P}_{3}|| z_{2}\right|^{2}+\left|z_{3}\right|^{2}<\varepsilon\left(\left|z_{0}\right|^{2}+\left|z_{1}\right|^{2}\right)\right\} .
$$

We note also $B_{\varepsilon}:=\partial V_{\varepsilon}$ the boundary of $V_{\varepsilon}$.

Since $X^{+}$is a attractor for $H$ there is an $\varepsilon>0$ such that

$$
X^{+} \subset V_{\varepsilon} \subset U^{+} \cup\left(\left\{z_{3}=0\right\} \backslash\{[0: 0: 1: 0]\}\right)
$$

and

$$
H\left(V_{\varepsilon}\right) \Subset V_{\varepsilon} .
$$

The mapping $p_{2}^{-1} \circ$ id $\circ p_{1}$ is biholomorphic onto its image in a open neighbourhood of $p_{1}^{-1}\left(B_{\varepsilon}\right) \subset W$ and therefore allows to identify holomorphically the two boundary components $p_{1}^{-1}\left(B_{\varepsilon}\right)$ and $p_{2}^{-1}\left(B_{\varepsilon}\right)$ of the complex space $A:=p_{2}^{-1}\left(V_{\varepsilon}\right) \backslash \overline{p_{1}^{-1}\left(V_{\varepsilon}\right)}$. This identification gives rise to a compact complex space $X$ with a global shell isomorphic $B$ to $B_{\varepsilon}$, i.e. $(X \backslash B)=A$ is connected. 
Let $N:=\operatorname{pr}_{1}^{-1}\left(V_{\varepsilon}\right)$ and $M:=\operatorname{pr}_{2}^{-1}\left(V_{\varepsilon}\right)$, i.e. $A=M \backslash N$. The following scheme illustrates the situation and the gluing up.

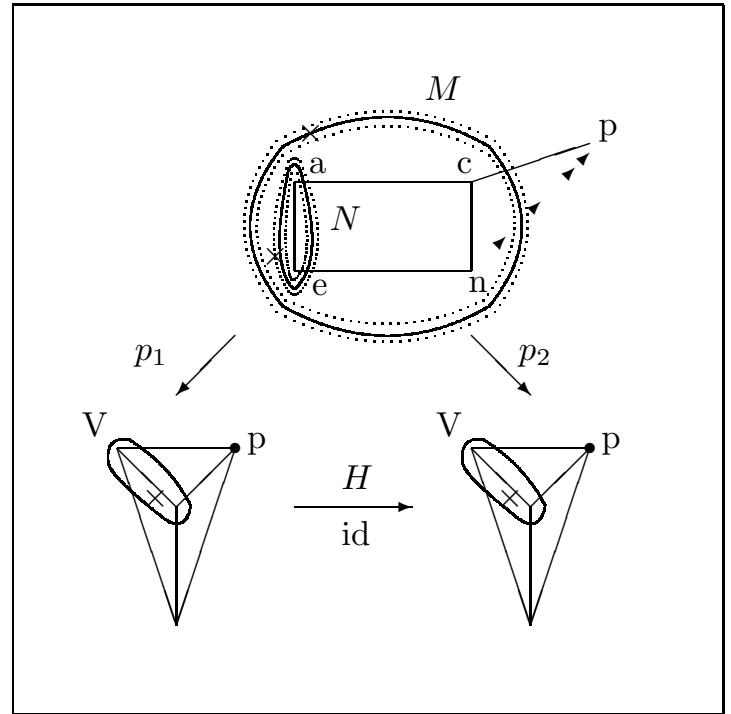

Figure 1. Gluing up.

The coordinates of the points are:

$$
\begin{aligned}
a & =[1: 0: 0: 0][1: 0: 0: 0] \\
n & =[0: 0: 1: 0][0: 1: 0: 0] \\
c & =[0: 0: 1: 0][1: 0: 0: 0] \\
p & =[0: 0: 1: 0][0: 0: 1: 0] \\
e & =[0: 1: 0: 0][0: 1: 0: 0] .
\end{aligned}
$$

By construction, the space $X$ is the union of the quotient $U^{+} /\langle H\rangle_{\mathbb{Z}}$ and of an irreducible divisor $D$ with $\pi_{1}(D)=\mathbb{Z}$. Furthermore, the space $X$ is singular exactly in the points corresponding to $l=D_{1} \cap D_{2}$. Of course it is possible to resolve the singularities by a finite sequence of modifications of $\mathbb{P}_{3} \times \mathbb{P}_{3}$ in order to obtain a smooth model of $X$. But since this process is not unique and does not change any of the properties discussed in the sequel, we shall work in what follows with the singular space. This space, and more generally all those that will be constructed 
in the same way, provide examples of class $L$, since there are non-singular rational curves in $B \subset X$ admitting an open neighbourhood isomorphic to $V$.

Let us remark that in [7], M. Kato classified all compact manifolds of class $L$ admitting a projective structure with fundamental group isomorphic to $\mathbb{Z}$. We shall see in what follows that our manifolds have this fundamental group. In view of Kato's result, it is easy to see that no smooth model of our spaces does admit any projective structure.

\section{Properties}

We shall use the same notations as in the previous paragraph.

Proposition 2.1. The fundamental group $\pi_{1}(X)$ is isomorphic to $\mathbb{Z}$.

Proof: Using the Mayer-Vietoris exact sequence, Van Kampen theorem and the fact that the space $A$ is simply connected, the proof works like in $[\mathbf{1}$, pp. 11-12].

We continue by calculating the fundamental group of the attracting basin $U^{+} \subset \mathbb{C}^{3}$ of $H$.

Proposition 2.2. $\pi_{1}\left(U^{+}\right)=1$.

Proof: Recall that

$$
V_{\varepsilon}=\left\{\varepsilon\left(\left|z_{0}\right|^{2}+\left|z_{1}\right|^{2}\right)>\left|z_{2}\right|^{2}+\left|z_{3}\right|^{2}\right\}
$$

in homogeneous coordinates $\left[z_{0}: z_{1}: z_{2}: z_{3}\right]$ in $\mathbb{P}_{3}$. It is evident that $V_{\varepsilon} \backslash\left\{z_{3}=0\right\}$ is simply connected.

Moreover, $U^{+}=\cup_{n \geq 0} H^{-n}\left(V_{\varepsilon} \backslash\left\{z_{3}=0\right\}\right)$. Let $\gamma: S^{1} \rightarrow U^{+}$be a closed curve with $K:=\gamma\left(S^{1}\right)$. There exists $n \in \mathbb{N}$ such as $K \subset H^{-n}\left(V_{\varepsilon} \backslash L\right)$ that is such that $H^{n}(K) \subset V_{\varepsilon} \backslash\left\{z_{3}=0\right\}$. Since $V_{\varepsilon} \backslash L$ is simply connected, we conclude that the curve $\gamma$ is trivial in $\pi_{1}\left(U^{+}\right)$.

Our next step is to give a lower bound of the complex dimension of $H^{1}(X, \mathcal{O})$.

Proposition 2.3. $\operatorname{dim}_{\mathbb{C}} H^{1}(X, \mathcal{O}) \geq 1$.

Proof: We shall show that $\operatorname{dim} H^{1}\left(X, \mathcal{O}^{*}\right)^{0} \geq 1$. To do so, we construct a non trivial twisted holmorphic line bundle. We denote by $\tilde{X}$ the universal cover of $X$ and by $\tilde{H}$ the generator of the fundamental group $\pi_{1}(X)$ induced by the initial automorphism $H$. Let us consider for $\lambda \neq 1$ the map:

$$
\begin{aligned}
\tilde{X} \times \mathbb{C} & \longrightarrow \tilde{X} \times \mathbb{C} \\
(x, z) & \longmapsto(\tilde{H}(x), \lambda z) .
\end{aligned}
$$


The projection onto the first component gives

$$
\tilde{X} \times \mathbb{C} / \mathbb{Z}=: L_{\lambda} \longrightarrow \tilde{X} / \mathbb{Z}=X
$$

The bundle $L_{\lambda}$ is a flat line bundle and therefore belongs to $H^{1}\left(X, \mathcal{O}^{*}\right)^{0}$, since $\pi_{1}(X)=\mathbb{Z}$. Suppose that there exists a non-trivial section $\sigma \in$ $H^{0}\left(X, L_{\lambda}\right)$. The section $\sigma$ induces a holomorphic function $f: \widetilde{X} \rightarrow \mathbb{C}$ such that $f(H(x))=\lambda f(x)$.

We know that $X=U^{+} \cup \widetilde{D} /\langle\widetilde{H}\rangle_{\mathbb{Z}}=\widetilde{X} /\langle\widetilde{H}\rangle_{\mathbb{Z}}$, where $\widetilde{D}$, the inverse image of the divisor $D$ in $\tilde{X}$, which is a connected infinite chaine of rational surfaces. Here we have noted by $\langle\widetilde{H}\rangle_{\mathbb{Z}}$ the group generated by the automorphism $\widetilde{H}$.

The function $f$ is necessarly equal to zero on the irreducible components of $\widetilde{D}$, hence on the whole divisor $\widetilde{D} \subset \widetilde{X}$ by connexity.

In what follows we shall see that there is a surface with global sperical shell (GSS) naturally associated to the space $X$.

For the sake of simplicity, we consider the case $c=0$, the other cases working analogously. Then the automorphism $H$ is given by

$$
H(x: y: z: t)=\left(x^{2}+t z: y^{2}+t x: t y: t^{2}\right) .
$$

The point $p=[1: 1: 0: 0]$ in $l=X^{+}$is a fixed point of saddle type. This is seen by an easy calculation: there are two eigenvalues equal to zero and one with absolute value strictly greater than one. At the point $p$, there exists locally a stable manifold noted by $W_{s}(p) \subset V_{\varepsilon}$ which is transversal to the attractor $l$, see $[8]$. Let $\mathcal{V}$ be a neighbourhood of $0 \in \mathbb{C}^{2}$ immersed onto the stable manifold. This gives rise to the commutative diagram:

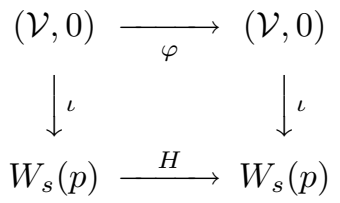

where $\iota$ denotes the immersion and $\varphi$ the induced selfmapping of $(\mathcal{V}, 0)$. 


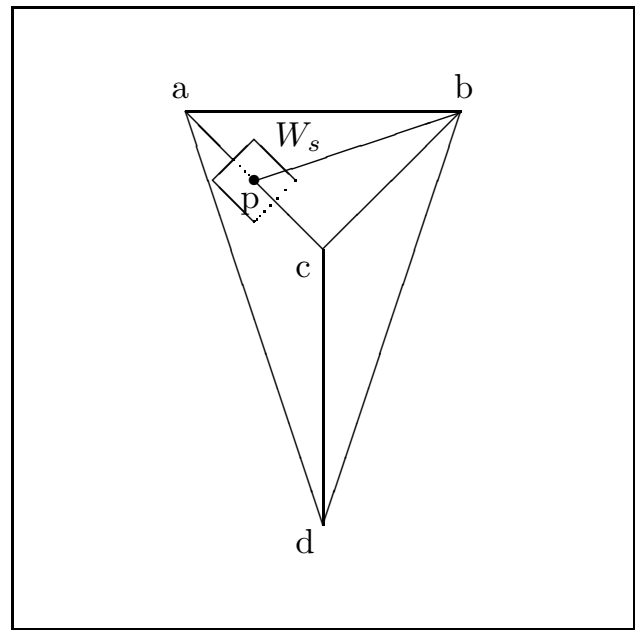

Figure 2. Stable manifold $W_{s}$.

Coordinates:

$$
\begin{aligned}
a & =[1: 0: 0: 0] \\
b & =[0: 0: 1: 0] \\
c & =[0: 1: 0: 0] \\
p & =[1: 1: 0: 0] \\
e & =[0: 0: 0: 1] .
\end{aligned}
$$

Let $C(\varphi)=\iota^{-1}(\iota(\mathcal{V}) \cap L)$ (where $L=\{t=0\}$ is the hyperplane at infinity) be the critical set of $\varphi$.

The restriction of $\varphi$ to $\mathcal{V} \backslash C(\varphi)$ is injective hence biholomorphic onto its image. Consequently, $\varphi$ is a strict germ of topological degree 1 . According to Ch. Favre [4], $\varphi$ is a Dloussky germ and thus defines a compact complex surface $S$ with global spherical shell.

Now the holomorphic function $f$ induces a holomorphic function $h:=$ $\iota \circ f$ on $\mathcal{V}$ with $h \circ \varphi=\lambda h$, which is zero on $C(\varphi)$. Since $\lambda \neq 1$, the function $h$ is identically zero on $\mathcal{V}$. This follows from the well-known fact that holomorphically non-trivial flat line bundles on surfaces with GSS have no non-trivial holomorphic sections, see e.g. [3].

We consider the increasing union

$$
Y:=\bigcup_{n \geq 0} H^{-n}\left(W_{s}(p) \backslash L\right) \subset U^{+},
$$


which is a 2-dimensional connected immersed submanifold of $U^{+}$and which is, as an abstract manifold, biholomorphic to the complement of the maximal divisor in the universal covering $\widetilde{S}$ of the above mentioned compact surface with GSS. Its topological closure in $\mathbb{P}_{3}(\mathbb{C})$ contains all the projective lines of the form $\{[x: y: t: 0] \mid t \in \mathbb{C}\}$, where $x / y \in S^{1}$ is a $2^{m}$-root of unity for some $m \in \mathbb{N}$. Therefore the quotient $S^{\prime}:=$ $Y /\langle H\rangle_{\mathbb{Z}} \subset X$ is not contained in any closed complex hypersurface of $X$. Since the function $h$ is identically zero, this is implies that the section $\sigma \in H^{0}\left(X, L_{\lambda}\right)$ vanishes identically, a contradiction to our assumption. We have proved

$$
\operatorname{dim} H^{1}(X, \mathcal{O}) \geq 1
$$

Now we show that there are no meromorphic functions on $X$.

Proposition 2.4. The algebraic dimension of the space $X$ is equal to zero.

Proof: We consider again $S^{\prime}:=Y /\langle H\rangle_{\mathbb{Z}} \subset X$ which is, as an abstract manifold biholomorphic to the complement of the maximal divisor in the GSS surface $S$. Let us suppose that there exists a meromorphic function $f$ on $X$. Then $f$ is constant on $S^{\prime}$ according to the properties of surfaces with global spherical shell. Since $S^{\prime}$ is not contained in any hypersurface in $X$, the function $f$ is constant on $X$. Therefore, the algebraic dimension $a(X)$ is equal to zero.

\section{Regular quadratic automorphisms allowing the construction}

The example with which we have worked for the moment is just a particular case of a regular quadratic automorphism of $\mathbb{C}^{3}$ having a projective line as an attractor at infinity; such automorphims belong to the fourth and fifth class in the classification of Fornæss-Wu. We shall give their list.

Those of the fourth class for which we can construct a compact complex space with global shell isomorphic to $B$ are of the form:

$$
H_{4}(x, y, z)=\left\{\begin{array}{l}
x^{2}+\alpha x y+\beta y^{2}+\delta y+\gamma+a z \\
y^{2}+\nu+x \\
y
\end{array}\right.
$$

the coefficients are chosen arbitrarily with $a \neq 0$. 
The inverse automorphism is of the form

$$
H_{4}^{-1}(x, y, z)=\left\{\begin{array}{l}
y-\nu-z^{2} \\
z \\
\frac{1}{a}(x-P(y, z)), \quad P \text { having } \operatorname{deg}_{z}(P)=4 .
\end{array}\right.
$$

These automorphisms admit as set of indeterminacy the point

$$
I^{+}=[0: 0: 1: 0]
$$

the inverse automorphisms the set $I^{-}=\{z=t=0\}$; we check immediately that $I^{+} \cap I^{-}=\emptyset$.

By the action of $H_{4}$, the hyperplane at infinity $\{t=0\}$ minus $I^{+}$is mapped to the $\mathbb{P}^{1}$ attractor $X^{+}=[x: y: 0: 0]$.

In order to get the topological closure of the graph in $\mathbb{P}_{3} \times \mathbb{P}_{3}$ one has to add the two divisors

$$
\begin{aligned}
D_{1}:=\left\{\left[z_{0}: z_{1}: z_{2}: 0\right],\left[z_{0}^{2}+\alpha z_{0} z_{1}+\beta z_{1}^{2}: z_{1}^{2}: 0: 0\right]\right\} \\
\cup\left\{[0: 0: 1: 0],\left[y_{0}: y_{1}: 0: 0\right]\right\} \\
D_{2}:=\left\{[0: 0: 1: 0],\left[y_{0}: y_{1}: y_{2}: 0\right]\right\} .
\end{aligned}
$$

As for the automorphisms of the fifth class, they are of the form:

$$
H_{5}(x, y, z)=\left\{\begin{array}{l}
y^{2}+\alpha x y+\beta x^{2}+\delta x+\gamma+a z \\
x^{2}+\theta+y \\
x
\end{array}\right.
$$

the constants are chosen arbitrarily with $a \neq 0$. The inverse automorphism is of the form

$$
H_{5}^{-1}(x, y, z)=\left\{\begin{array}{l}
z \\
y-\theta-z^{2} \\
\frac{1}{a}(x-P(y, z)), \quad P \text { having } \operatorname{deg}_{z}(P)=4 .
\end{array}\right.
$$

These automorphisms admit as the indetermination set the point

$$
I^{+}=[0: 0: 1: 0]
$$

as for the inverse automorphisms the set $I^{-}=\{z=t=0\}$; it is again clear that $I^{+} \cap I^{-}=\emptyset$ and that $H_{5}$ maps the hyperplane at infinity $\{t=0\}$ minus $I^{+}$to the $\mathbb{P}^{1}$ attractor $X^{+}=[x: y: 0: 0]$. 
Furthermore, for the topological closure of the graph in $\mathbb{P}_{3} \times \mathbb{P}_{3}$ one adds

$$
\begin{aligned}
D_{1}:=\left\{\left[z_{0}: z_{1}: z_{2}: 0\right],\left[z_{1}^{2}+\alpha z_{0} z_{1}+\beta z_{0}^{2}: z_{0} 2: 0: 0\right]\right\} \\
\cup\left\{[0: 0: 1: 0],\left[y_{0}: y_{1}: 0: 0\right]\right\} \\
D_{2}:=\left\{[0: 0: 1: 0],\left[y_{0}: y_{1}: y_{2}: 0\right]\right\}
\end{aligned}
$$

\section{Conclusion}

We gather the different results in:

Theorem 4.1. The regular quadratic automorphisms of $\mathbb{C}^{3}$ in the list of Fornass-Wu [5] from which one can construct a compact complex threedimensional space of class $L$ with a global spherical shell isomorphic to $B$ are:

(1) In the fourth class, those of the form

$$
H_{4}(x, y, z)= \begin{cases}x^{2}+\alpha x y+\beta y^{2}+\delta y+\gamma+a z & \\ y^{2}+\nu+x, & a \neq 0 \\ y & \end{cases}
$$

(2) In the fifth class, those of the form

$$
H_{5}(x, y, z)= \begin{cases}y^{2}+\alpha x y+\beta x^{2}+\delta x+\gamma+a z & \\ x^{2}+\theta+y, & a \neq 0 \\ x . & \end{cases}
$$

The space $X$ has the following properties:

- the fundamental group is $\pi_{1}(X)=\mathbb{Z}$,

- the fundamental group of the attraction basin in $\mathbb{C}^{3}$ is: $\pi_{1}\left(U^{+}\right)=1$,

- $\operatorname{dim} H^{1}(X, \mathcal{O}) \geq 1$,

- the algebraic dimension is equal to zero: $a(X)=0$.

\section{References}

[1] G. Dloussky, Structure des surfaces de Kato, Mém. Soc. Math. France (N.S.) 14 (1984), 120 pp.

[2] G. Dloussky and K. Oelueklaus, Surfaces de la classe $\mathrm{VII}_{0}$ et automorphismes de Hénon, C. R. Acad. Sci. Paris Sér. I Math. 328(7) (1999), 609-612. 
[3] G. Dloussky and K. Oelueklaus, Vector fields and foliations on compact surfaces of class $\mathrm{VII}_{0}$, Ann. Inst. Fourier (Grenoble) 49(5) (1999), 1503-1545.

[4] Ch. FAvre, Classification of 2-dimensional contracting rigid germs and Kato surfaces. I, J. Math. Pures Appl. (9) 79(5) (2000), 475-514.

[5] J. E. Fornæss AND H. Wu, Classification of degree 2 polynomial automorphisms of $C^{3}$, Publ. Mat. 42(1) (1998), 195-210.

[6] M. KATO, On compact complex 3-folds with lines, Japan. J. Math. (N.S.) 11(1) (1985), 1-58.

[7] M. KATO, Compact complex 3-folds with projective structures: the infinite cyclic fundamental group case, Saitama Math. J. 4 (1986), 35-49.

[8] N. Sibony, Dynamique des applications rationnelles de $\mathbf{P}^{k}$, in: " $D y$ namique et géométrie complexes" (Lyon, 1997), Panor. Synthèses 8, Soc. Math. France, Paris, 1999, pp. ix-x, xi-xii, 97-185.

LATP-UMR(CNRS) 6632

CMI-Université d'Aix-Marseille I

39 , rue Joliot-Curie

F-13453 Marseille Cedex 13

France

E-mail address: karloelj@cmi.univ-mrs.fr

E-mail address: renaud@cmi.univ-mrs.fr

Primera versió rebuda el 6 de setembre de 2005, darrera versió rebuda el 19 de gener de 2006. 